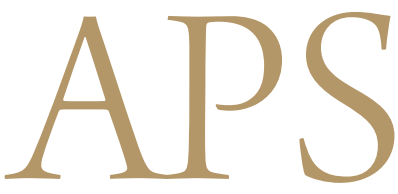

Archives of Plastic Surgery

\title{
A Comparative Study of CG CryoDerm and AlloDerm in Direct-to-Implant Immediate Breast Reconstruction
}

\author{
Jun Ho Lee ${ }^{1}$, Ki Rin Park ${ }^{1}$, Tae Gon $\mathrm{Kim}^{1}{ }^{1}$ Ju-Ho Ha ${ }^{1}$, Kyu-Jin Chung ${ }^{1}$, Yong-Ha Kim ${ }^{1}$, \\ Soo Jung Lee ${ }^{2}$, Soo Hwan Kang ${ }^{2}$ \\ Departments of ${ }^{1}$ Plastic and Reconstructive Surgery and ${ }^{2}$ General Surgery, Yeungnam University College of Medicine, Daegu, Korea
}

Background To date, various types of acellular dermal matrix (ADM) have been developed for clinical use. AlloDerm is the most familiar type of ADM to most surgeons in breast reconstruction. It is prepared by freeze-drying. CG CryoDerm is the first form of ADM that requires no drying process. Therefore, theoretically, it has a higher degree of preservation of the dermal structures than AlloDerm. We conducted this study to compare the clinical course and postoperative outcomes of patients who underwent direct-to-implant breast reconstructions using AlloDerm and those who did using CG CryoDerm.

Methods We performed a retrospective analysis of the medical records in a consecutive series of 50 patients who underwent direct-to-implant breast reconstruction using AlloDerm $(n=31)$ or CryoDerm $(n=19)$. We then compared the clinical course and postoperative outcomes of the two groups based on the overall incidence of complications and the duration of drainage. Results The mean follow-up period was 16 months. There were no significant differences in the overall incidence of complications (seroma, infection, skin flap necrosis, capsular contracture, and implant loss) between the two groups. Nor was there any significant difference in the duration of drainage.

Conclusions CG CryoDerm has the merits of short preparation time and easy handling during surgery. Our results indicate that CG CryoDerm might be an alternative allograft material to AlloDerm in direct-to-implant breast reconstruction.

\section{Keywords Mammaplasty / Breast implantation / Acellular dermis}

Correspondence: Jun Ho Lee Department of Plastic and Reconstructive Surgery, Yeungnam University Hospital, Yeungnam University College of Medicine, 170 Hyeonchung-ro, Nam-gu, Daegu 705-717, Korea

Tel: +82-53-620-3482

Fax: +82-53-626-0705

E-mail: psjhlee@naver.com

This article was presented at the 70th Congress of the Korean Society of Plastic and Reconstructive Surgeons on November 9, 2012 in Seoul, Korea.

No potential conflict of interest relevant to this article was reported.

Received: 15 Mar 2013 • Revised: 26 Apr 2013 • Accepted: 12 May 2013

pISSN: 2234-6163• elSSN: 2234-6171 • http://dx.doi.org/10.5999/aps.2013.40.4.374• Arch Plast Surg 2013;40:374-379

\section{INTRODUCTION}

Over the past several years, with various advancements and innovations, there have been changes in the traditional concept of breast reconstruction. The use of acellular dermal matrix (ADM) in breast reconstruction is one of these changes that has had a significant impact on breast reconstruction [1-3].
$\mathrm{ADM}$ is a biodegradable tissue material with regenerative potential, and it is capable of revascularization and recellularization [4]. Thus, surgeons can optimally position an implant or a tissue expander on the chest wall, facilitating one- and two-stage reconstruction. $\mathrm{ADM}$ can also minimize periprosthetic fibrosis and appears to lessen the inflammatory response associated with prosthetic devices [5]. 
There are many different types of ADM that are currently available for clinical use. However, none contain exactly the same materials. Moreover, many studies have reported that the inherent biomechanical properties of the products differ among the types of $\mathrm{ADM}$, leading to different outcomes $[6,7]$. It is therefore mandatory to clearly understand the biological properties of ADM that vary based on the methods of processing and packaging because they are directly associated with its incorporation into the tissue.

AlloDerm is the most familiar ADM to most surgeons. It was first used as an inferolateral sling for breast reconstruction in 2005 [1]. It is prepared by freeze-drying. On the other hand, once CG CryoDerm is frozen, it requires no drying process. Therefore, from a theoretical perspective, it has a higher degree of preservation of the dermal structures than does AlloDerm.

This is the first study to compare the clinical course and postoperative outcomes of patients who underwent direct-to-implant breast reconstructions using AlloDerm and those who did using CG CryoDerm.

\section{METHODS}

In the current study, we performed a retrospective review of the medical records of patients who underwent direct-to-implant immediate breast reconstruction using AlloDerm (LifeCell Corp., Branchburg, NJ, USA) or CG CryoDerm (CGBio Corp., Seongnam, Korea). All of the breast reconstructions were performed by a single plastic surgeon. We used AlloDerm during a period ranging from June of 2010 to May of 2012 and CG

\section{Fig. 1. Acellular dermal matrix after preparation}

The preparation of (A) AlloDerm and (B) CG CryoDerm. It takes less time to prepare CG CryoDerm than AlloDerm because CG CryoDerm can be melted but needs no rehydration.
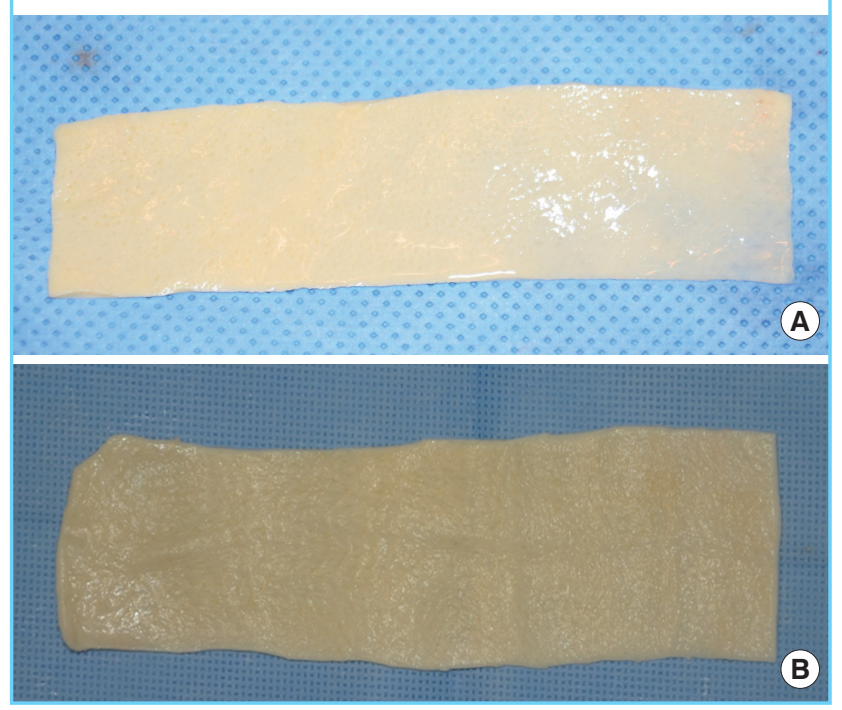

CryoDerm from October of 2011 to April of 2012. From 2011 to 2012, we randomly selected either of the two types of ADM to cover the implant inferolaterally. The exclusion criteria for the current study were as follows: 1) a history of undergoing irradiation before and after reconstruction, 2) a history of undergoing neoadjuvant chemotherapy as part of breast cancer treatment, and 3) a history of undergoing reconstructive surgery.

We collected baseline data such as demographic information, the clinical course, and postoperative outcomes. All of the patients underwent direct-to-implant immediate breast reconstruction with one of the two types of $\mathrm{ADM}$ and Biocell textured silicone gel implants (Allergan Corp., Irvine, CA, USA). We divided our clinical series of patients into two groups: the AlloDerm group and the CG CryoDerm group. Each group underwent breast reconstruction with a $4 \mathrm{~cm} \times 12 \mathrm{~cm}$ sheet of AlloDerm with a thickness of 2.31 to $3.30 \mathrm{~mm}$ or a $4 \mathrm{~cm} \times 14$ $\mathrm{cm}$ sheet of CG CryoDerm with a thickness of 1.04 to $2.29 \mathrm{~mm}$ (Fig. 1). After skin sparing mastectomy or nipple-areolar sparing mastectomy, an implant was then inserted into the newly created submuscular pocket. The implant was covered superiorly with the pectoralis muscle and inferolaterally with an ADM sling and serratus fascia (Fig. 2). In a patient with a large breast size requiring a large implant such as $400 \mathrm{~mL}$, the $\mathrm{ADM}$ could be insufficient to cover the implant inferolaterally. In this situation, both inferior edges of the ADM that remained after covering the implant inferolaterally were trimmed and transposed to the required shape and dimension.

We evaluated the clinical course and postoperative outcomes based on the following factors: 1) seroma, 2) infection, 3) hematoma, 4) skin flap necrosis, 5) capsular contracture (Baker grade III or IV), 6) loss of implant, and 7) duration of drainage.

Statistical analysis was performed using SPSS (SPSS, Inc.,

\section{Fig. 2. Intraoperative photograph of acellular dermal matrix}

Intraoperative photograph of direct-to-implant immediate breast reconstruction with acellular dermal matrix. CG CryoDerm is used as coverage of the prosthesis at the lower pole of the breast.

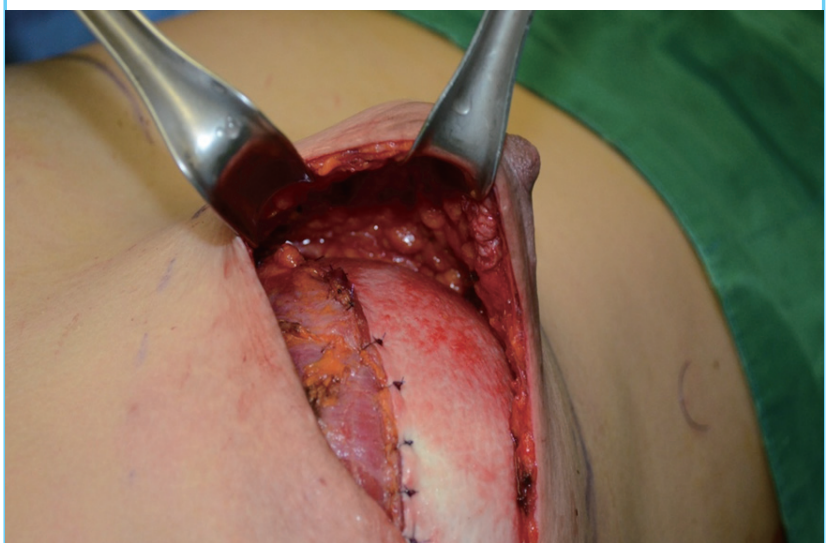


Chicago, IL, USA). To compare the patient characteristics (age, body mass index, implant size) and mean duration of drainage, we performed an independent $\mathrm{t}$-test. We performed a chisquared test to compare the patient characteristics (adjuvant chemotherapy and skin incision) and Fisher's exact test to compare the overall incidence of complications and breast cancer stage of the two groups. The complications of the two groups were compared based on odds ratios and confidence intervals. A P-value of $<0.05$ was considered statistically significant.

\section{RESULTS}

A total of 31 patients who underwent direct-to-implant immediate breast reconstruction with AlloDerm and 19 patients who

\section{Table 1. Patient characteristics}

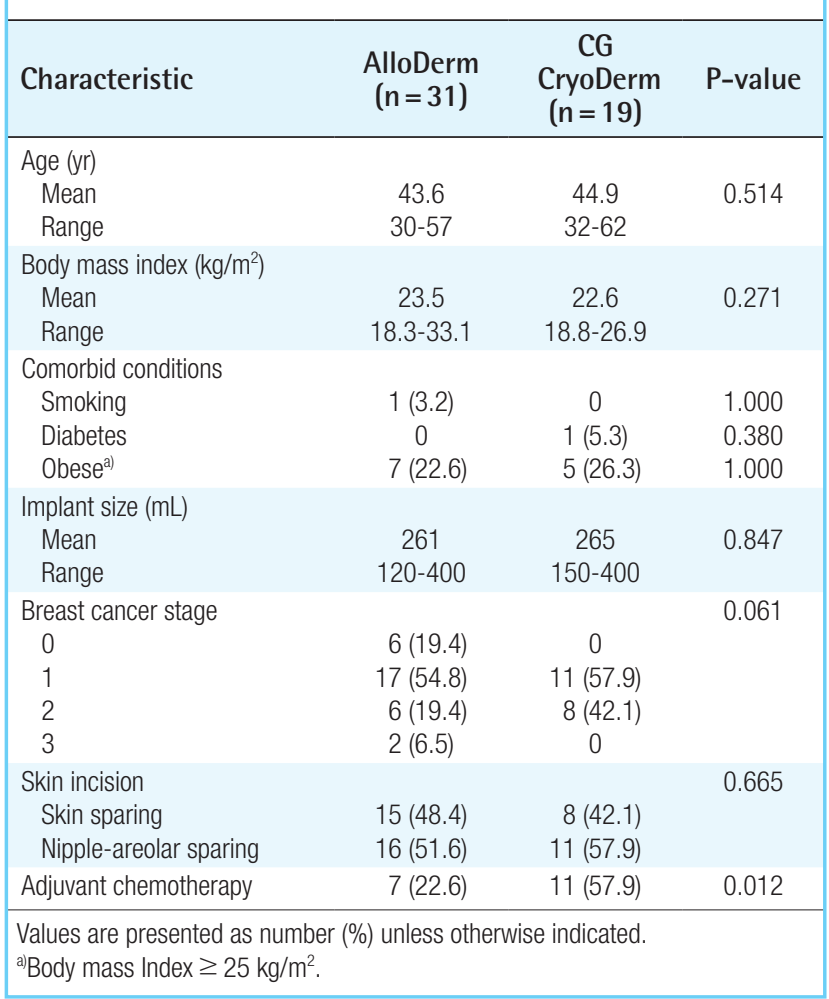

underwent reconstruction with CG CryoDerm were identified. We therefore assigned them to the AlloDerm group $(\mathrm{n}=31)$ and the CG CryoDerm group $(n=19)$. The mean follow-up period was 17 months in the AlloDerm group and 14 months in the CG CryoDerm group.

There were no significant differences in the demographic and clinical data between the two groups, except for the fact that the frequency of adjuvant chemotherapy was higher in the CG CryoDerm group (Table 1). There were one patient in the AlloDerm group who had a smoking history and one patient in the CG CryoDerm group who had a history of diabetes. Approximately $25 \%$ of the patients of both groups were obese.

There was no significant difference in the overall incidence of complications between the two groups (Table 2). In the AlloDerm group, the overall incidence of complications was $22.6 \%$ (7/31); these included five cases (16.1\%) of seroma, four cases (12.9\%) of infection (defined as a condition where the use of intravenous antibiotics was needed), one case (3.2\%) of skin flap necrosis, and one case (3.2\%) of capsular contracture (Baker grade III or IV). One case (3.2\%) of implant loss was secondary to skin flap necrosis followed by uncontrolled infection. In the CG CryoDerm group, the overall incidence of complications was $26.3 \%$ (5/19); these included one case (5.3\%) of seroma, four cases $(21.1 \%)$ of infection, and one case (5.3\%) of capsular contracture (Baker grade III/IV). One case (5.3\%) of implant loss secondary to implant rupture at 1 year after surgery was reconstructed with autologous tissue.

In all of the patients, closed-suction drains were used and then discontinued when drainage was less than $20 \mathrm{~mL}$ over 24 hours for two consecutive days. The mean duration of drainage was

\begin{tabular}{|lccc|}
\hline Table 3. Duration of drainage & & \\
\hline Drainage & $\begin{array}{c}\text { AlloDerm } \\
(\%)\end{array}$ & $\begin{array}{c}\text { CG CryoDerm } \\
(\%)\end{array}$ & P-value \\
\hline Duration of drainage (day) & 14.2 & 14.3 & 0.947 \\
Mean & $6-27$ & $10-28$ & \\
Range & & & \\
\hline
\end{tabular}

Table 2. Postoperative complications between AlloDerm- or CG CryoDerm-assisted direct-to-implant breast reconstruction

\begin{tabular}{|c|c|c|c|c|}
\hline Complication & $\begin{array}{l}\text { AlloDerm }(\%) \\
\quad(n=31)\end{array}$ & $\begin{array}{c}\text { CG CryoDerm }(\%) \\
(n=19)\end{array}$ & $\begin{array}{c}\text { Odds ratio } \\
\text { (95\% confidence interval) }\end{array}$ & P-value \\
\hline Total complications ${ }^{\mathrm{a})}$ & $7(22.6)$ & $5(26.3)$ & $1.22(0.32-4.60)$ & 0.764 \\
\hline Seroma & $5(16.1)$ & $1(5.3)$ & $0.29(0.03-2.69)$ & 0.387 \\
\hline Infection & $4(12.9)$ & $4(21.1)$ & $1.80(0.39-8.25)$ & 0.459 \\
\hline Hematoma & 0 & 0 & - & \\
\hline Skin flap necrosis & $1(3.2)$ & 0 & - & 1.000 \\
\hline Capsular contracture (Baker grade III or IV) & $1(3.2)$ & $1(5.3)$ & $1.67(0.10-28.32)$ & 1.000 \\
\hline Implant loss & $1(3.2)$ & $1(5.3)$ & $1.67(0.10-28.32)$ & 1.000 \\
\hline
\end{tabular}


14.2 days in the AlloDerm group and 14.3 days in the CG CryoDerm group $(\mathrm{P}=0.947)$ (Table 3$)$.

\section{DISCUSSION}

$\mathrm{ADM}$ is a biotechnological tissue prepared from either human or animal skin that has gained widespread acceptance for use in breast reconstruction [1-3], abdominal hernia repair [8,9], pelvic reconstruction $[10,11]$, and head and neck contouring and reconstruction [12-16]. During the manufacturing process, the cellular components that might cause rejection and inflammation are removed. Thus, it is possible to produce a structurally intact tissue matrix that serves as the biological scaffold that is necessary for tissue ingrowth, angiogenesis, and ultimately, tissue regeneration $[2,17,18]$. After mastectomy, the ADM can be used to create the inferolateral portion of the tissue expander pocket (two-stage reconstruction) or implant pocket (direct-toimplant reconstruction) [2].

To date, various types of tissue products have been developed for breast reconstruction, and these include AlloDerm (LifeCell Corp.) [17], Strattice (LifeCell Corp.) [19], DermaMatrix (Synthes Inc., West Chester, PA, USA) [20], FlexHD (Ethicon Inc., Somerville, NJ, USA) [21], and Permacol (Covidien, Mansfield, MA, USA) [22]. In addition, these products vary in many ways, depending on the source of tissue material, manufacturing methods, storage and surgical preparation, available size, and cost. However, their use is often limited due to the lack of outcome data.

CG CryoDerm is the first $\mathrm{ADM}$ that is prepared using the freezing method, and it was introduced in 2010. To date, however, no published studies have reported its use in breast reconstruction. CG CryoDerm should be frozen at a temperature of $-40^{\circ} \mathrm{C}$, and it has a shelf-life of five years. It takes less than 3 minutes to prepare CG CryoDerm as compared with more than 15 minutes for AlloDerm because CG CryoDerm can be melted but needs no rehydration. It is terminally sterile and requires an orientation. In freeze-drying an ADM such as AlloDerm, ice crystals might be generated during the freezing process. This causes the primary destruction of the tissues. In addition, in the subsequent drying process, as the water evaporates and the structure is deformed, space is created. Once the CG CryoDerm is frozen, however, it requires no drying process. Therefore, it has a higher degree of preservation of the dermal structures. In addition, from a theoretical perspective, it also has a greater tensile strength, a higher elasticity, a lower biodegradability, and a lower incidence of inflammation as compared with freeze-dried ADM. Furthermore, there are also differences between the two products in the methods of handling during surgery. In our experience, the CG CryoDerm appears to be more pliable and elastic than AlloDerm. Thus, this made it easier to shape the sheet to the inferolateral curvature of the breast.

Our results showed that the use of AlloDerm and CG CryoDerm are both effective procedures, with a similar incidence of complications, in supporting the position of the implant in its original location and creating the inferior and lateral folds of the breast. Theoretically, the technique of preparation of CG CryoDerm should cause less destruction to the normal dermal tissue than that of Alloderm. We have therefore speculated that the treatment outcomes would be better in the CG CryoDerm group than the AlloDerm group. Based on such findings as seroma, infection, hematoma, skin flap necrosis, capsular contracture, implant loss, and the duration of drainage, however, there were no significant differences in the clinical course or postoperative outcomes between the two groups. These results suggest that, considering the equivalence of their characteristics, the choice between these two products is subject to the judgment of surgeons.

In the current study, seroma and infection have a higher incidence than other complications in both of the two groups. Seroma could be effectively managed with a closed drainage system. We placed two drains in different planes. One drain was placed between the skin flap and the ADM layer, and a second drain was placed between the implant and the $\mathrm{ADM}$ layer. The drains were left in place until there was less than $20 \mathrm{~mL}$ of drainage over a 24-hour period, which could take 3 weeks. Though there were no significant differences among the two groups, the CG CryoDerm group had a tendency toward a higher infection rate than the AlloDerm group (Fig. 3). All infection cases were local infection or cellulitis, which was resolved through a course

\section{Fig. 3. Infected breast after adjuvant chemotherapy \\ A case of a 62-year-old woman with an infected left breast 1 month after direct-to-implant immediate breast reconstruction with CG CryoDerm. Note the erythema in the lower portion of the left breast. This cellulitis was resolved through a course of intravenous antibiotics.}

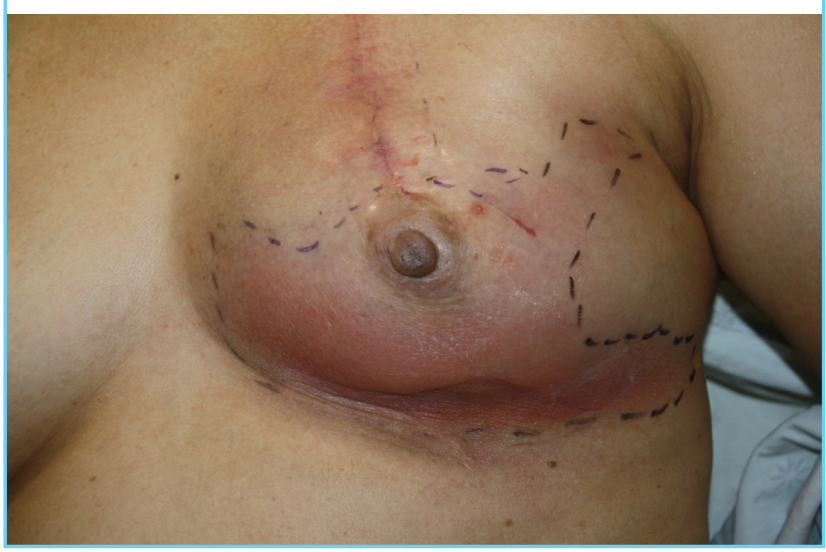


of either oral or intravenous antibiotics, except one case of the AlloDerm group. There were more patients taking adjuvant chemotherapy after mastectomy in the CG CryoDerm group than the AlloDerm group, which could have influenced the observed outcome.

There are some limitations of the current study as follows: 1 ) We enrolled a small number of patients and they each had their own individual problems, which might be underpowered. 2) We failed to determine whether there were any differences in the graft compliance and the degree of incorporation or neovascularization between the two groups. This is because we did not obtain tissue samples from the sites of the dermal graft for histologic examination in one-stage breast reconstruction. Further large-scale studies are therefore warranted to confirm the efficacy of CG CryoDerm on histologic examination in patients who underwent direct-to-implant immediate breast reconstruction.

To summarize, there were no significant differences in the overall incidence of complications or the duration of drainage between patients who underwent direct-to-implant breast reconstructions using AlloDerm and those who did using CG CryoDerm. In conclusion, our results indicate that CG CryoDerm might be an alternative allograft material to AlloDerm in directto-implant breast reconstruction. CG CryoDerm has the merit of short preparation time and easy handling during surgery.

\section{REFERENCES}

1. Breuing KH, Warren SM. Immediate bilateral breast reconstruction with implants and inferolateral AlloDerm slings. Ann Plast Surg 2005;55:232-9.

2. Spear SL, Pelletiere SC, Lockwood M. Immediate breast reconstruction with tissue expanders and AlloDerm. In: Spear SL, Willey SC, Robb GL, et al., editors. Surgery of the breast: principles and art. 2nd ed. Philadelphia: Lippincott Williams \& Wilkins; 2006. p.484-8.

3. Salzberg CA. Direct-to-implant breast reconstruction. Clin Plast Surg 2012;39:119-26.

4. Cheng A, Saint-Cyr M. Comparison of different ADM materials in breast surgery. Clin Plast Surg 2012;39:167-75.

5. Nahabedian MY. Acellular dermal matrices in primary breast reconstruction: principles, concepts, and indications. Plast Reconstr Surg 2012;130:44S-53S.

6. Gabriel A, Maxwell GP. Evolving role of alloderm in breast surgery. Plast Surg Nurs 2011;31:141-50.

7. Basu CB, Leong M, Hicks MJ. Acellular cadaveric dermis decreases the inflammatory response in capsule formation in reconstructive breast surgery. Plast Reconstr Surg 2010;126:1842-7.
8. Buinewicz B, Rosen B. Acellular cadaveric dermis (AlloDerm): a new alternative for abdominal hernia repair. Ann Plast Surg 2004;52:188-94.

9. Butler CE, Prieto VG. Reduction of adhesions with composite AlloDerm/polypropylene mesh implants for abdominal wall reconstruction. Plast Reconstr Surg 2004;114:464-73.

10. Butler CE, Langstein HN, Kronowitz SJ. Pelvic, abdominal, and chest wall reconstruction with AlloDerm in patients at increased risk for mesh-related complications. Plast Reconstr Surg 2005;116:1263-75.

11. Clemons JL, Myers DL, Aguilar VC, et al. Vaginal paravaginal repair with an AlloDerm graft. Am J Obstet Gynecol 2003; 189:1612-8.

12. Rohrich RJ, Reagan BJ, Adams WP Jr, et al. Early results of vermilion lip augmentation using acellular allogeneic dermis: an adjunct in facial rejuvenation. Plast Reconstr Surg 2000;105:409-16.

13. Jackson IT, Yavuzer R. AlloDerm for dorsal nasal irregularities. Plast Reconstr Surg 2001;107:553-8.

14. Sinha UK, Saadat D, Doherty CM, et al. Use of AlloDerm implant to prevent frey syndrome after parotidectomy. Arch Facial Plast Surg 2003;5:109-12.

15. Taban M, Douglas R, Li T, et al. Efficacy of "thick" acellular human dermis (AlloDerm) for lower eyelid reconstruction: comparison with hard palate and thin AlloDerm grafts. Arch Facial Plast Surg 2005; 7:38-44.

16. Kim HS, Suh HW, Ha KY, et al. The usefulness of the endonasal incisional approach for the treatment of nasal bone fracture. Arch Plast Surg 2012;39:209-15.

17. Lifecell. AlloDerm tissue matrix defined [Internet]. Branchburg, NJ: LifeCell Corp.; 2013 [cited 2013 Mar 8]. Available from: http://www.lifecell.com/health-care-professionals/ lifecell-products/allodermr-regenerative-tissue-matrix/ allodermr-tissue-matrix-defined.

18. Breuing KH, Colwell AS. Inferolateral AlloDerm hammock for implant coverage in breast reconstruction. Ann Plast Surg 2007;59:250-5.

19. Lifecell. Strattice reconstructive tissue matrix [Internet]. Branchburg, NJ: LifeCell Corp.; 2013 [cited 2013 Mar 8]. Available from: http://www.lifecell.com/health-careprofessionals/lifecell-products/stratticetm-reconstructivetissue-matrix.

20. Synthes. DermaMatrix acellular dermis [Internet]. Philadelphia: Synthes Inc.; 2012 [cited 2013 Mar 8]. Available from: http://www.synthes.com/sites/NA/Products/CMF/AcellularDermis/Pages/DermaMatrix_Acellular_Dermis.aspx.

21. Ethicon Inc. FlexHD acellular hydrated dermis for breast reconstruction [Internet]. New Jersey: Ethicon Inc.; 2013 [cit- 
ed 2013 Mar 8]. Available from: http://www.ethicon360. com/products/flex-hd-acel-hydrated-dermis-breast-reconstruction.

22. Covidien. Permacol surgical implant [Internet]. Mansfield:
Covidien; 2013 [cited 2013 Mar 8]. Available from: http:// www.autosuture.com/autosuture/pagebuilder.aspx?webPag eID $=0 \&$ topicID $=170603 \& x s l=x s l /$ productPagePrint.xsl. 